\title{
NOUVELLES ESPECES ET NOUVELLES LOCALISATIONS \\ DE CULICOIDES (DIPTERA : CERATOPOGONIDAE) \\ DES ARDENNES, DU CENTRE DE LA FRANCE, DU JURA FRANÇAIS ET DU JURA SUISSE
}

\author{
Par J. CALLOT, M. KREMER et Y. DEDUIT
}

Sauf deux, toutes les espèces de Culicoides que nous signalerons au cours de cette note ont été obtenues à partir des gîtes larvaires. A propos de chacune d'elles, nous indiquerons leurs localisations géographiques. Les prélèvements du Jura sont de septembre 1961, ceux des Ardennes de juin 1961, ceux de Seine-et-Marne et de l'Aisne sont de mai 1961.

Les espèces se rangent dans les groupes suivants :

GROUPE «pulicaris \$: aile claire à taches sombres, deuxième cellule radiale en partie entamée par une tache sombre. Thorax diversement orné.

Hypopygium mâle à processus petits, en crochets ; paramères poilus à leur extrémité. Apodèmes ventraux courts, souvent en crochet. Bosse poilue sur la face interne du coxite. Femelle ayant deux spermathèques. Pas de sensilles sur les articles 4 à 10 des antennes. Troisième article du palpe à nombreuses dépressions sensorielles. Yeux généralement soudés.

GROUPE « fascipennis »: aile légèrement ombrée avec des taches claires de part et d'autre de la deuxième cellule radiale (celle-ci n'étant pas entamée par ces taches claires).

Hypopygium mâle à lamelle généralement profondément entaillée. Aedeagus trapézoïdal. Membrane basale spiculée.

Femelle : deux spermathèques. Pas de sensilles sur les articles 4 à 10 ; yeux séparés.

GROuPE * odibilis »: ailes grises ou brunes à taches claires. Des taches à l'extrémité des ailes et dans les diverses cellules, la seconde cellule radiale étant pourtant entièrement sombre, en général.

Hypopygium mâle à lamelle modérément entaillée. Processus digitiformes et longs. Membrane nue ou spiculée. Paramères plus ou moins effilés à leur partie distale.

Femelles : deux spermathèques. Des sensilles sur tous les articles des antennes (sauf chez certaines espèces). Troisième article du palpe à dépression sensorielle unique. Yeux séparés. 
Groupe \& cubitalis \$: ailes comme dans le groupe fascipennis.

Hypopygiums mâles et particularités des femelles analogues à celles du groupe odibilis.

GROUPE « sphagnumensis »: ailes souvent semblables à celles du groupe odibilis, mais souvent dépourvues de taches à leur extrémité distale.

Hypopygium mâle à lamelle peu entaillée, souvent convexe. Processus petits. Aedeagus triangulaire. Pas d'apodèmes ventraux. Membrane basale toujours dépourvue de spicules.

Femelles: une seule spermathèque. Yeux séparés. Troisième article du palpe à fossette sensorielle unique. Des sensilles sur tous les articles des antennes. Indice antennaire au voisinage de 2,00.

Groupe « obsoletus 》: ailes à taches grises et claires, deuxième cellule radiale en partie entamée par une tache claire.

Hypopygium mâle à lamelle dépourvue de processus ou à processus rudimentaires. Pas de sensilles sur les articles 4 à 10 des antennes des femelles. Deux spermathèques.

GROUPE «stigma-nubeculosus »: hypopygium mâle à paramères soudés en leur milieu. Aedeagus généralement bifide.

Femelles: une spermathèque. Des sensilles seulement sur les articles antennaires 3 et de 8 à 10, parfois également sur 6 et 7 . Yeux séparés.

Catégorie «stigma $»$ : aile banche avec une seule tache sur la deuxième cellule radiale.

Catégorie «nubecolosus » : ailes d'aspect nuageux à taches blanches et grises.

\section{Groupe pulicaris}

On peut distinguer dans le groupe « pulicaris », d'une façon non absolue, les espèces ayant une tache sombre dans la cellule cubitale et celles qui en sont dépourvues. Celles qui nous intéressent dans cette étude appartiennent à la première catégorie. C. punctatus (Meig.) se distingue aisément des autres, grâce à la présence d'ocelles claires dans les taches sombres de l'extrémité des nervures M1, M2 et souvent également Cu1. De plus, la tache en sablier de l'extrémité de la cellule r5 présente une brisure de son angle inféro-externe. $C$. pulicaris et $C$. lupicaris n'ont pas d'ocelles et leur tache en sablier est entière. Dans la description originale de lupicaris, nous pouvons relever les points suivants : tache sombre du bord postérieur de la cellule anale accolée à ce bord (elle en est séparée par une bande claire chez pulicaris). Dessins du thorax bien plus étendus que chez pulicaris.

Campbell et Pelham-Clinton ajoutent les détails suivants: indice antennaire ne dépassant généralement pas 1,05 et jamais 1,1 pour $C$. pulicaris; indice antennaire généralement supérieur à 1,05 et souvent supérieur à 1,1 pour C. lupicaris (et $C$. delta). 
Chez le premier, les dessins du thorax sont variables, mais la pointe médiane est relativement courte en arrière, par rapport aux deux latérales; chez le second, les dessins sont toujours importants et la pointe médiane est plus longue par rapport à celles des côtés.

Aucun de ces caractères ne donne, en fait, une parfaite certitude. Les indices de $C$. pulicaris sont parfois légèrement supérieurs à ceux donnés par Campbell et Pelham-Clinton. Les taches du thorax de cette espèce prennent parfois un développement important et la pointe médiane peut être relativement plus longue, comme chez $C$. lupicaris.

Nous avons considéré, comme des $C$. lupicaris, ceux qui présentent des taches thoraciques très importantes, une tache sombre de la cellule anale touchant son bord postérieur, et qui ont un indice égal ou supérieur à 1,9 . Nous rangeons dans l'espèce pulicaris les exemplaires ne répondant pas à ces trois critères.

CULICOIDES PULICARIS (L.).

Ardennes: La Férée (source de la Serre et marnière dans les bois de La Férée).

Ardennes : Regniowez (sous le pont de la Loge-Rosette).

CULICOIDES PUNCTATUS (Meig.).

Aisne: marais de Chivres.

Ardennes: La Férée (marnière dans le bois de La Férée) ; Signy-le-Petit (étang de la Motte).

Jura : lac de Chambly (500 m.).

Doubs : La Petite-Chaux (La Chenoy, prairie humide) ; Le Grand-Pin-sur-leLac (tourbière, $1.100 \mathrm{~m}$.) ; Brey-et-Maison-du-Bois (tourbière, $925 \mathrm{~m}$.).

Canton de Vaud (Suisse): Combe-des-Amburnex (bord d'un ruisseau, $1.300 \mathrm{~m}$.).

CULICOIDES LUPICARIS Downes et Kettle.

Ardennes: La Férée (marnière dans les bois de La Férée).

\section{Groupe fascipennis}

CULICOIDES FASCIPENNIS Staeger.

Doubs: lac de Belle-Fontaine.

CULICOIDES PALLIDICORNIS Kieffer.

Canton de Vaud (Suisse): Combe-des-Amburnex.

Aisne : route de Pierrepont à Velles (eaux stagnantes).

Ardennes: La Férée (source de la Serre).

\section{Groupe odibilis}

CULICOIDES ODIBILIS Austen.

Doubs : Grand-Pin-sur-le-Lac (tourbière, $1.100 \mathrm{~m}$.) ; Mouthe (terrains tour- 
beux à la source du Doubs, $950 \mathrm{~m}$.) ; La Chapelle-des-Bois (mare boueuse, sur un chemin, $1.100 \mathrm{~m}$.) ; Les Granges-Ste-Marie (marais : gîte à Aedes cataphylla, $900 \mathrm{~m}$.).

Jura : Chemenot (berge d'un étang, $200 \mathrm{~m}$.).

Canton de Vaud (Suisse) : Combe-des-Amburnex (1.300 m.).

Haute-Saône : Faucogney (étang près des Guidons, $400 \mathrm{~m}$.).

Ardennes: Signy-le-Petit (berge de l'étang de la Motte; fossé de drainage en forêt) ; Blanchefosse (berge de la mare des Grands-Caullaux) ; Tarzy (berge près du pont des Socquettes); La Férée (marnière en forêt).

Ardennes (Belgique): étang de Nimellette (berge).

Seine-et-Marne : Courpalay (boues sous le pont de l'Yvron).

\section{CULICOIDES MUSILATOR Krémer et Callot.}

Rappelons que cette espèce se distingue de $C$. simulator par les points suivants : thorax jaune à raies longitudinales bien marquées. Apodème ventral du mâle plus long et fin. Indice antennaire des femelles supérieur en général à 1,65 , toujours supérieur à 1,56 .

Seine-et-Marne: Courpalay (boues sous le pont de l'Yvron).

\section{CULICOIDES CLASTRIERI n. sp.}

Espèce de taille moyenne. Thorax de couleur jaune brunâtre et abdomen vert comme chez odibilis. Ornementation du thorax réduite à la fossette préscutale et aux deux taches habituelles situées en avant d'elle. Parfois, présence d'un trait médian sur la moitié antérieure et très rarement ébauche de deux traits latéraux.

- Ailes analogues à celles de $C$. odibilis dans leur ensemble. Cependant, la tache distale de la cellule $r 5$ est très allongée, à peine séparée de la précédente située après la seconde cellule radiale.

Peigne tibial postérieur formé de quatre grandes soies dont les longueurs sont : $57 / 64,5 / 55,1 / 45,6 \mu$.

Mâle.

Tête : yeux se touchant.

Article du palpe cylindrique. Troisième article portant une fossette sensorielle très petite. Longueurs des articles : $57 / 45,6 / 32,3$ et $36,1 \mu$ (deux premiers articles comptés ensemble).

Antennes: premier article claviforme, les suivants légèrement ovalaires, à col aminci, leur moitié proximale est claire et la moitié distale est sombre ; douzième article présentant un rétrécissement dans son tiers distal; treizième cylindrique, sauf à sa base qui est renflée ; quatorzième parfaitement cylindrique ; quinzième légèrement lancéolé.

Longueur des articles: 91/41,8/41,8/41,8/38/38/38/38/38/47,5/140,6/ $100,7 / 117,8 \mu$. 
Ailes: taches claires plus importantes que chez la femelle. Macrotriches assez abondantes, respectant les cellules sous-costales et basales.

Longueur : $1,12 \mathrm{~mm}$. ; largeur : $0,48 \mathrm{~mm}$. ; costa : 0,59 mm. (compté à partir de l'arculus).

Hypopygium mâle: tout à fait identique à celui de $C$. odibilis. Lamelle modérément entaillée. Processus digitiformes et divergents. Sternite assez profondément entaillé en courbe douce. Membrane basale nue. Aedeagus à bras fortement chitinisés et se prolongeant à l'intérieur du corps, jusqu'au bord distal de celui-ci. Corps en forme d'écusson renversé et, à part les prolongements des bras, il est assez peu chitinisé.

Coxite assez long ; apodème ventral long, assez épais à sa base, mais allant en s'amincissant. Apodème dorsal en forme de chaussure. Style légèrement renflé dans sa partie distale.

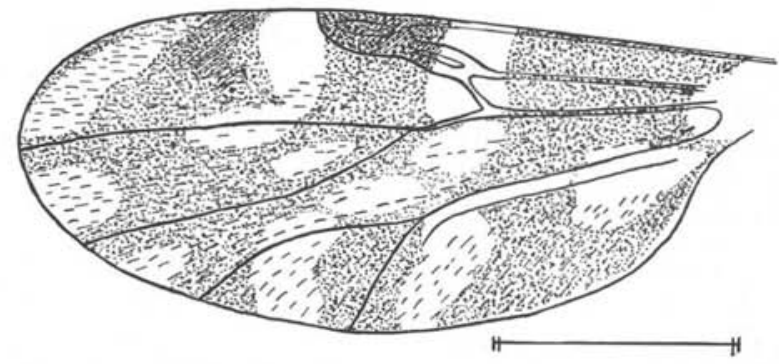

Fig. 1. - Culicoides clastrieri n. sp. Aile de la femelle (Le trait égale $0,5 \mathrm{~mm}$.)

\section{Femelle.}

Tête : yeux séparés par une zone assez étroite, réunis par un trait au-dessus du poil médian.

Palpe : deux premiers articles cylindriques allongés, le troisième plus globuleux et présentant une fossette unique et large.

Longueur des articles: 91,2/68,4/36,1/32,3 $\mu$ (deux premiers articles comptés ensemble).

Antennes : premier article claviforme, les suivants de plus en plus allongés, en forme de tonneaux. Articles 11 à 14 cylindriques, avec une base plus renflée. Dernier article allongé, en forme de cigare ou lancéolé. Il n’y a pas de sensilles sur les articles 4 à 10 inclus. Ce caractère permet de discuter l'appartenance de cette espèce au groupe odibilis.

Longueur des articles 3-15: 49,4/30,4/32,3/34,2/34,2/34,2/34,2/36,1/ $72,2 / 76 / 83,6 / 83,6 / 104,5 \mu$.

Nombre de sensilles: $8,0,0,0,0,0,0,0,2,2,2,3,3$.

Indice antennaire : 1,47 (variant entre 1,38 et 1,53 selon les exemplaires).

Ailes: taches plus rondes et moins étendues que chez le mâle. Macrotriches 
plus abondantes, mais respectant les mêmes cellules. Longueur : 1,28 mm. ; largeur : $0,60 \mathrm{~mm}$; costa : $0,78 \mathrm{~mm}$.

Spermathèques: deux fonctionnelles à peu près sphériques, présentant un petit col, dont les longueurs sont 38 et $45,6 \mu$; une spermathèque rudimentaire de $11,5 \mu$.

Ce qui frappe immédiatement chez $C$. clastrieri, c'est sa similitude avec C. odibilis. Les hypopygiums mâles ne présentent pratiquement pas de différences. Mais le thorax de $C$. odibilis est orné de vigoureux dessins qui peuvent, il est vrai, être fortement diminués chez certains exemplaires. La forme de la tache distale de r5 de $C$. clastrieri est différente de celle de $C$. odibilis, de même que de celle de $C$. neavei Austen. Les mâles de cette dernière espècé peuvent encore être distingués de $C$. clastrieri par l'absence d'apodème ventral, comme il ressort du dessin de Carter, Ingram et Macfie.
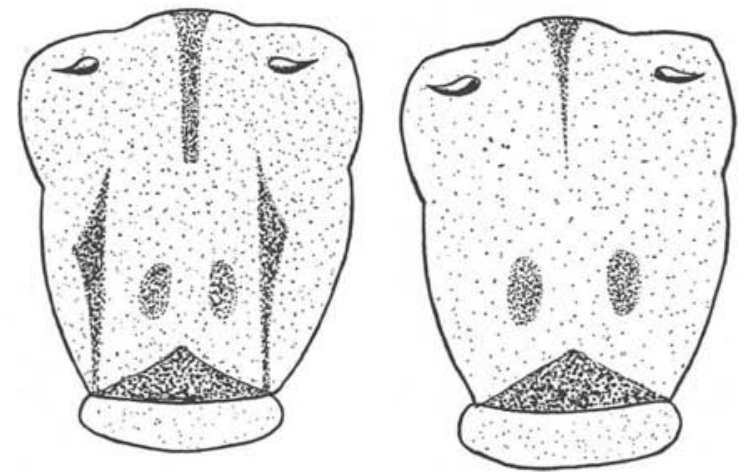

FIG. 2. - Culicoides clastrieri n. sp.

Thorax de la femelle : deux types

L'absence de sensilles sur les articles 4 à 10 des femelles permet de distinguer et peut-être mêmé d'éxclure $C$. clastrieri du groupe odibilis, comme il ressort de sa description générale. La présence d'une tache claire dans la base de $\mathrm{m} 1$ permet d'exclure les espèces qui én sont dépourvues, telles $C$. simulator, musilator, haranti, begueti.

Nous avions proposé dans un précédent article de mettre en synonymie $C$. odibilis et $C$. pictimargo, et c'était peut-être une erreur. Il nous paraît préférable d'admettre actuellement l'existence de trois espècés ayant des hypopygiums mâles tout à fait identiques: $C$. odibilis, $C$. pictimargo et $C$. clastrieri.

C. odibilis : thorax fortement orné, des sensilles sur tous les articles antennaires de la femelle.

C. pictimargo: thorax pratiquement dépourvu de dessins, les sensilles sont absents sur les articles 11 et 12 . (L'exemplaire de $C$. odibilis que nous avions 
signalé comme dépourvu de sensilles sur les articles 11 et 12 doit probablement être considéré comme présentant une anomalie, que nous avons depuis constatée également chez d'autres espèces).

C. clastrieri: thorax pratiquement dépourvu de dessins, la tache claire de l'extrémité de la cellule r5 bien plus importante que dans les espèces précédentes. Il n'y a pas de sensilles sur les articles 4 à 10 de la femelle.
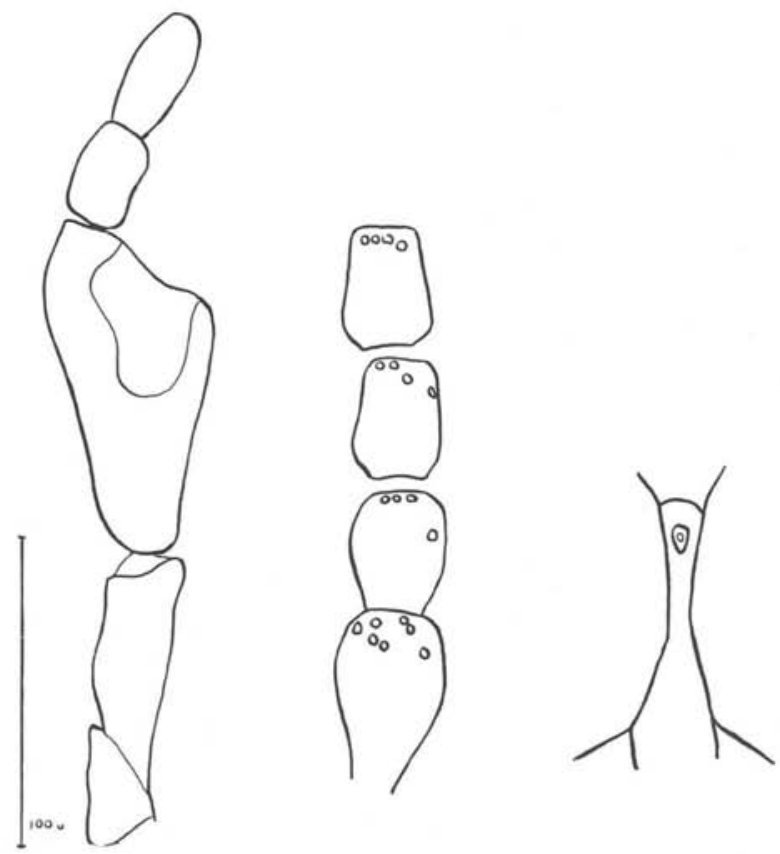

Fic. 3. - Culicoides clastrieri n. sp. Palpe; premiers articles antennaires et espace inter-oculaire de la femelle.

C. clastrieri a été trouvé dans les gîtes suivants :

Haut-Rhin: Guémar (bord de fossé de drainage) : holotype.

Bas-Rhin : Krautergersheim (bord de fossé) : allotype (1).

Seine-et-Marne : Courpalay (boues sur le bord de l'Yvron).

Ardennes: un mâle a été obtenu de l'étang de la Motte à Signy-le-Petit, mais il s'agit peut-être d'un Culicoides odibilis à dessins du thorax entièrement régressés.

Cette espèce est dédiée à $\mathrm{M}$. le Docteur J. Clastrier, Chef de Service à l'Institut Pasteur d'Alger.

(1) Ces prélèvements de la plaine du Rhin sont de mai 1961. 


\section{Groupe cubitalis}

\section{CULICOIDES CUBITALIS Edwards.}

Aisne : route de Pierrepont à Villes (eaux stagnantes), marais de Pierrepont (boues sur une digue), marais de Chivres (canal de drainage).

Doubs : Les Granges-Ste-Marie (Les Champs-Nouveaux, marécage à Aedes cataphylla), Vaux-et-Chantegrue (tourbière).

Canton de Vaud (Suisse) : Combe-des-Amburnex (bord de ruisseau dans un pré, $1.300 \mathrm{~m}$.).

\section{CULICOIDES JURENSIS n. sp.}

Espèce de petite taille, proche de $C$. cubitalis. Thorax brunâtre, dépourvu de taches, scutellum légèrement plus clair que le scutum. Ailes gris très clair. On y distingue avec peine une petite tache claire après la deuxième cellule radiale (1) et sur la partie proximale de la première. Macrotriches assez abondantes, mais absentes dans les cellules sous-costales, basales et à la base de l'aile.

Peigne tibial formé de quatre soies dont les rapports sont : 13/13/11,5/11,5.

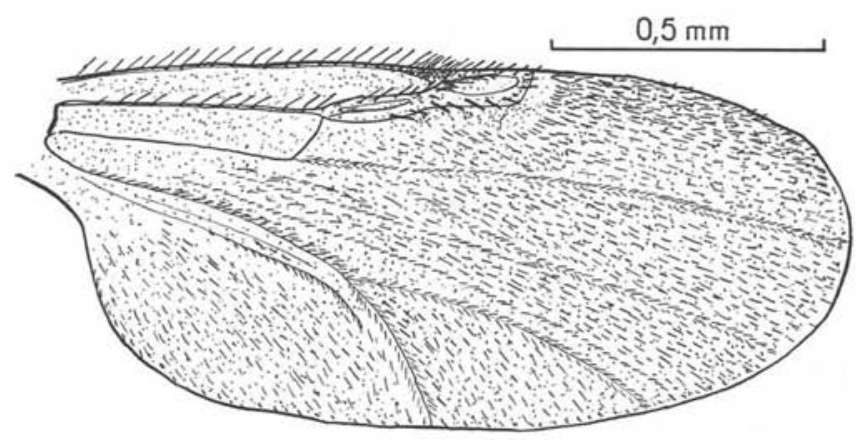

Fig. 4. - Culicoides jurensis n. sp. Aile de la femelle

Mâle.

Tête : yeux se touchant. Premier article des antennes claviforme, les suivants plus ou moins sphériques, à col rétréci, de chaque côté, et insertion des verticelles médiane. Douzième article à col distal allongé, insertion des verticelles sur le tiers proximal. Treizième et quatorzième articles plus ou moins cylindriques, à base renflée. Dernier article lancéolé.

Longueur des articles 3 à $15: 91,2 / 38 / 38 / 38 / 38 / 38 / 41,8 / 38 / 41,8 / 57 /$ 140,6/114/119,7 $\mu$.

Palpe : premiers articles cylindriques. Troisième légèrement renflé dans son tiers distal, avec une fossette sensorielle assez petite.

(1) Ces taches semblent plutôt dues à une absence de macrotriches qu'à une véritable dépigmentation. 
Longueur des articles: 72,2/72,2/36,1/41,8 $\mu$.

Ailes: macrotriches peu abondantes. Longueur: 1,45 mm. ; largeur : $0,55 \mathrm{~mm}$. ; costa : $0,78 \mathrm{~mm}$.

Hypopygium : lamelle modérément entaillée. Processus digitiformes et divergents. Sternite profondément et largement entaillé. Membrane basale spiculée. Aedeagus trapézoïdal et peu chitinisé. Bras de l'aedeagus fortement chitinisés et recourbés à leur origine. Paramères renflés en triangle à leur base, formant rapidement ensuite un angle, puis s'amincissant progressivement; l'extrémité seule est recourbée. Apodème ventral du coxite long et fin, apodème corsal digitiforme. Coxite de forme habituelle, style renflé dans sa partie distale.

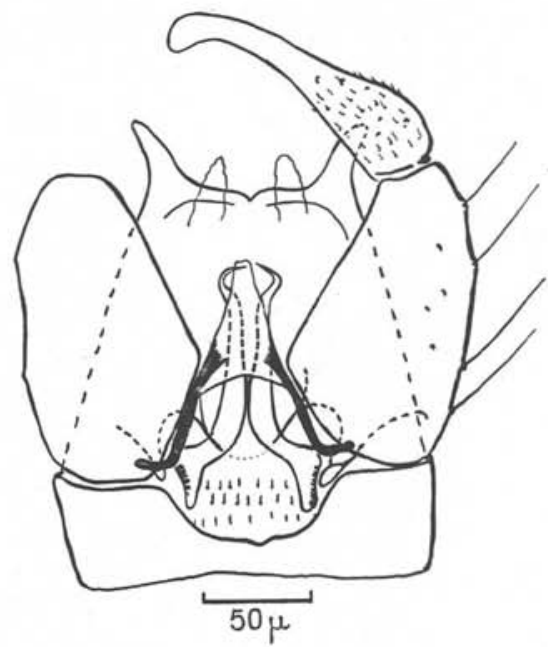

FIG. 5. - Culicoides jurensis n. sp. Hypopygium du mâle

\section{Femelle.}

Yeux séparés.

Longueur des articles antennaires 3 à $15: 51,3 / 39,1 / 41,8 / 41,8 / 41,8 / 41,8 /$ $41,8 / 41,8 / 66,1 / 66,5 / 74,1 / 76 / 98,8 \mu$.

Indice du type 1,11 .

Répartition des sensilles (moyenne des deux antennes du type) : 7,5/5,5/ $4 / 4 / 3,5 / 3,5 / 3 / 3 / 1,5 / 2 / 2 / 3 / 1$.

Troisième article du palpe bien renflé et présentant une fossette sensorielle unique et très profonde.

Longueur des articles: 89,3/114/30,4/41,8 $\mu$ (deux premiers articles comptés ensemble).

Ann. de Parasitologie, T. XXXVII, No $1-2 .-1962$. 
Ailes : macrotriches bien plus abondantes que chez le mâle, mais respectant les mêmes cellules. Longueur : 1,51 mm. ; largeur : 0,69 mm. ; costa : 0,87 mm. (comptés à partir de l'arculus).

Spermathèques: deux fonctionnelles, ovoïdes, à petit col, de 53 et $49,5 \mu$. Spermathèque rudimentaire ovoïde, allongée, de 12,5 $\mu$.

L'aspect de l'hypopygium et la disposition des sensilles chez les femelles permettent de séparer aisément $C$. jurensis des espèces du groupe fascipennis,

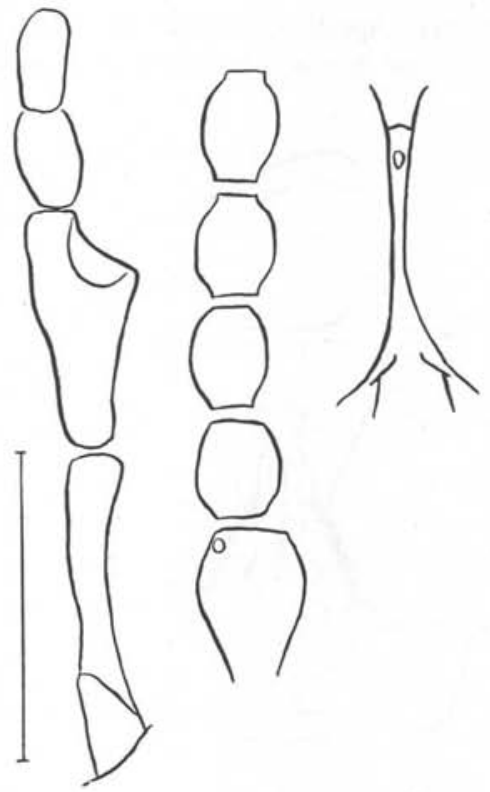

Fig. 6. - Culicoides jurensis n. sp. Palpe ; premiers articles antennaires et espace inter-oculairc de la femelle (Même échelle que fig. 8).

auquel l'apparentent ses ailes. La spiculation de la membrane basale ne se retrouve pas chez les espèces $C$. cubitalis, $C$. begueti, $C$. pseudo-heliophilus et C. gambiae, avec lesquelles les mâles de jurensis présentent l'un ou l'autre caractère en commun. La présence de sensilles sur tous les articles des antennes permet de différencier les femelles de celles de $C$. begueti, $C$. semimaculatus. L'indice anténnairé de $C$. cubitalis est bien plus élevé que celui de $C$. jurensis. La longueur des ailes de $C$. susae Kieffer, espèce mal connue, est plus petite que celle de $C$. jurénsis. La femelle de $C$. gambiae est très difficile à distinguer de cette espèce ; le nombre total de ses sensilles est pourtant beaucoup moins élevé. 
L'aspect des ailes distingue aisément cette espèce des autres représentants paléarctiques du groupe « odibilis ».

Doubs: La Chapelle-des-Bois (bord de mare près d'un gouffre) : 1 \%, 2 , holotype et allotype.

Nous avons également trouvé un mâle dans un prélèvement de boues d'un fossé de drainage, dans la plaine d'Alsace, près de Guémar (Haut-Rhin).

\section{Groupe sphagnumensis}

Nous avons, dans un récent article sur la faune des Hautes-Vosges, tenté de délimiter le problème posé par le complexe $C$. sphagnumensis-C. carjalaensis. Les exemplaires que nous avons obtenus dans les régions qui nous intéressent iui correspondent à la variété $C$. sphagnumensis vogesicus, différente du type par la présence de taches claires dans la partie distale des cellules r $5, \mathrm{~m} 1$ et $\mathrm{m} 2$ et de $C$. carjalaensis par l'absence de macrotriches dans la cellule basale.

CULICOIDES SPHAGNUMENSIS Williams var. VOGESICUS Krémer et Callot.

Jura : Bois-d'Amont (tourbière).

Haute-Saône: étang de Foucogney.

Vosges: près du Thillot (tourbière).

C. sphagnumensis vogesicus et $C$. carjalaensis sont par ailleurs très fréquents dans tous les terrains tourbeux des hauts sommets des Vosges (département du Haut-Rhin).

\section{Groupe obsoletus}

CULICOIDES OBSOLETUS Meigen.

Seine-et-Marne: Courpalay : deux femelles capturées à la lumière.

\section{Groupe stigma-riethi}

\section{CULICOIDES RIETHI Kieffer.}

Cette espèce appartient à la catégorie de celles ayant, comme $C$. nubeculosus, des ailes d'un aspect nuageux.

Une femelle fut capturée par l'un de nous à la lumière (à Courpalay), en même temps que $C$. obsoletus. Il nous est impossible actuellement d'expliquer la présence de cette espèze halophile, facile à reconnaître, dans cette région, où les gîtes salés sont inconnus. Signalons à ce propos qu'il nous est arrivé de capturer au piège lumineux à Strasbourg d'autres espèces typiquement halophiles, alors que les gîtes que nous avons recensés dans l'Est sont, pour les plus proches, situés à $90 \mathrm{~km}$. 


\section{CULICOIDES HELVETICUS n. sp.}

\section{Femelle.}

Espèce de taille moyenne à ailes présentant une tache unique. Ailes blanches, à l'exception de la deuxième cellule radiale et la partie distale de la première dont les nervures sont plus foncées, de même que les zones qu'elles délimitent. Longueur des ailes: 1,42 mm. ; largeur: 0,63 mm. ; costa : $0,82 \mathrm{~mm}$. (comptés à partir de l'arculus).

Thorax assez voisin de celui de $C$. parroti. Couleur de fond grise. Une bande brun sombre part du bord antérieur, s'élargit progressivement jusqu'au deuxième tiers du ssutum, où elle se termine. Sur le tiers moyen, deux bandes

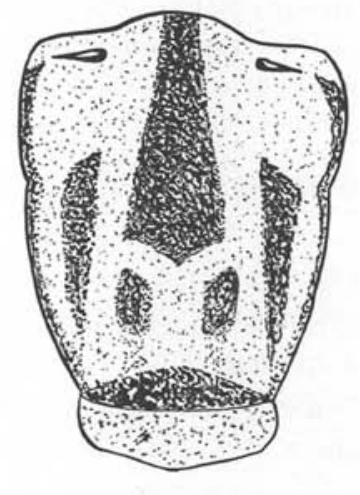

Fig. 7. - Culicoides helveticus n. sp. Thoras de la femelle.

sombres latérales. A la limite latérale, deux autres bandes sombres festonnées. Fossette préscutale et taches rondes situées en avant, également sombres. Scutellum du même gris que le scutum avec parfois une bande médiane sombre. Absence de petites taches grises comme chez parroti.

Tête : yeux très largement séparés.

Premier article flagellum claviforme, mais court et, de ce fait, presque piriforme. Article suivant en forme de tonneaux quelque peu évasés distalement. Onzième article un peu renflé dans sa partie proximale, évasé distalement. Articles suivants plus étroits. Quinzième article lancéolé ou en forme de cigare.

Longueur des articles: 49/38/35,3/38/36/36,1/37,6/38/53,2/53,2/60,8/ $66,8 / 95,7 \mu$.

Indice antennaire : 1,07 (compris entre 1,02 et 1,12 pour les cotypes).

Disposition des sensilles: $1 / 0 / 0 / 1 / 1 / 2 / 2 / 2,5 / 0 / 0 / 0 / 0 / 0$ (moyenne des deux antennes du type). 

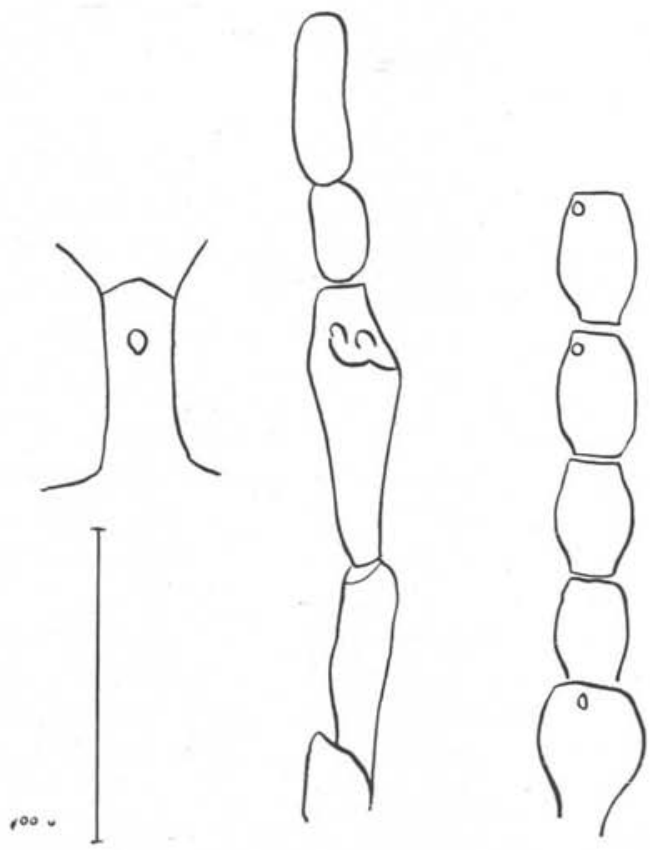

FIG. 8. - Culicoides helveticus n. sp. Palpe ; premiers articles antennaires et espace interoculaire de la femelle.

On a pu constater chez deux exemplaires, mais sur une des deux antennes seulement, l'absence des sensilles sur les articles 3, 6 et 7 . Un exemplaire possède d'un côté deux sensilles sur le $7^{\mathrm{e}}$ article. Troisième article du palpe à peine renflé au-delà de son milieu où se trouve une grande fossette sensorielle ; de plus, présence au mêmé nivéau de trois à quatre petites fossettes sensorielles.

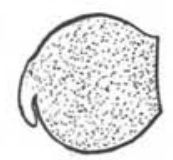
FIG. 9. - Culicoides helve- ticus femelle. Spermathè- que.

Longueur des articles : 83,6/87,4/38/42,9 $\mu$ (deux premiers articles comptés ensemble).

Mandibules et maxilles munies de dents. 
Pattes: peigne tibial postérieur avec cinq soies dont les longueurs sont: $49,4 / 57 / 41,8 / 41,8 / 32,3 \mu$. Chez certains exemplaires, les deux premières épines sont de tailles identiques ; chez certains, la troisième est un peu plus longue que la quatrième.

Spermathèque: une spermathèque unique à peu près sphérique, dont le diamètre atteint $45,6 \mu$. Pas de col chitinisé. Du côté opposé à l'ouverture, présence d'un petit réceptacle en forme de doigt de gant recourbé, comme chez C. stigma.

\section{Mâle.}

Un exemplaire est éclos après la rédaction de ce travail. Il diffère de C. stigma et de C. stigmoides par l'écartement plus important des deux pointes du corps de l'ædeagus; sa membrane basale est nue (alors qu'elle est spiculée chez $C$. stigmoides) ; le coxite n'est pas renflé à sa base comme chez C. stigma , le sternite n'est pas échancré. Il n'y a pas de points sombres sur le thorax comme chez $C$. parroti.

Par la forme de la spermathèque, $C$. helveticus est très voisin de $C$. stigma. Les dessins du thorax, mis à part l'absence de taches punctiformes, rappellent ceux de $C$. parroti. La présence de sensilles sur les articles 6 et 7 est tout à fait originale dans le groupe stigma. Le thorax permet de distinguer les femelles de celles des espèces stigma et stigmoides.

Canton de Vaud (Suisse): Combe des Amburnex (bas-fond de la Combe : 3 : holotype ; idem. au bord d'un gouffre: 1 \%).
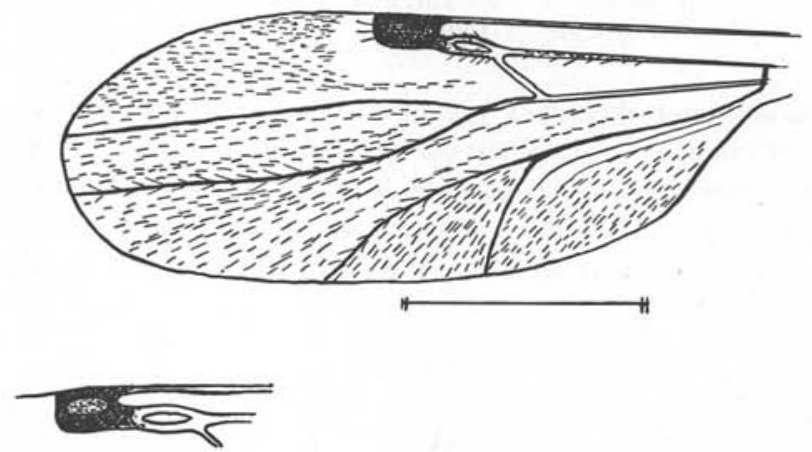

Fig. 10. - Culicoides stigmoides n. sp. Aile de la femel'e et détail des cellules radiales (même échelle que fig. 1)

\section{CULICOIDES STIGMOIDES n. sp.}

Nous avons eu l'occasion de signaler à plusieurs reprises la présence dans l'Est de la France de C. stigma (Mg.). Nous étions cependant frappés par le fait que la membrane basale des hypopygiums mâles était toujours spiculée 
alors que le schéma d'Edwards montrait une membrane nue. Nous pensions à une omission, mais des textes plus récents nous ont amené à modifier ce point de vue et à décrire ici $C$. stigmoides différent de $C$. stigma essentiellement par sa membrane spiculée.

Ailes blanches à l'exception de la deuxième cellule radiale et de la partie toute distale de la première qui sont de couleur noire. Parfois, seules les nervures sont noires et l'espace qu'elles délimitent est plus clair.

Thorax brun-noir. Les deux taches préscutales sont placées dans un carré plus clair et forment une croix de Malte.

Mâle.

Yeux soudés.

Longueur des articles antennaires: 76/41,8/39,9/41/41,8/38/37,2/38/ $34,2 / 49,4 / 125,4 / 91,2 / 102,6 \mu$.

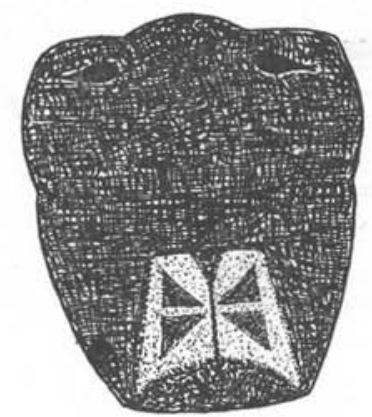

FIG. 11. - Culicoides stigmoides n. sp. Thorax de la femelle.

Des sensilles sur les articles 8,9 et 10 . Premier article claviforme, les suivants sphériques, avec insertions des poils en-deça du milieu. Treizième article renflé à sa base puis cylindrique, quatorzième cylindrique, quinzième en cigare ou lancéolé.

Longueur des articles du palpe : 64,6/64,6/38/51,3 $\mu$ (deux premiers comptés ensemble).

Longueur des ailes: 1,26 mm. ; largeur : 0,46 mm. Costa : 0,69. Hypopygium. Aspect général identique à celui de $C$. stigma. Lamelle bien entaillée. Présence de deux lobes internes par rapport au processus et placés de part et d'autre de la fente de la lamelle. Ces lobes, moins grands que chez $C$. stigma, d'aspect variable, parfois très petits, parfois formant même un $3^{\circ}$ lobe supplémentaire. Sternite profondément et largement échancré. Membrane basale abondamment spiculée. Paramères soudés par leur base qui forme une sorte d'arche. Bord postérieur de cette arche plus fortement chitinisé. A cette arche 
font suite deux pointes dirigées vers l'arrière. Aedeagus bifide dans sa partie distale. Coxite assez trapu, sans renflement distal analogue à celui de stigma . Apodème ventral court et mousse. Style sans aucun renflement dans sa partie distale.
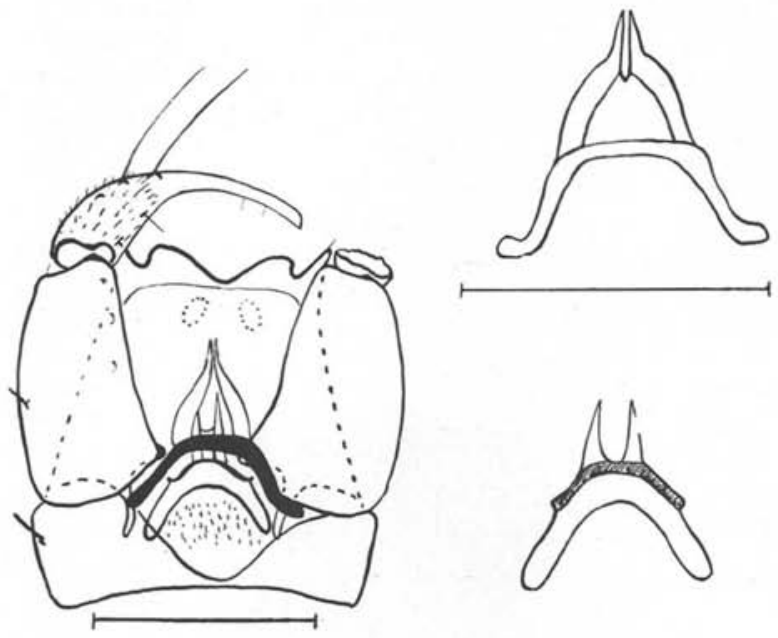

F1G. 12. -Culicoides stigmoides n. sp. Hypopygium du mâle ; aedeagus ; paramères (Même échelle que fig. 8)

\section{Femelle.}

Yeux largement écartés, réunis par un trait brisé au-dessus du poil médian. Souvent, présence d'une suture médiane partant de cette suture frontale.

\section{Antennes :}

Premier article claviforme, les suivants en forme de tonneaux. Les cinq derniers plus ou moins lancéolés ou en fût de canon.

Longueur des articles : 49,4/38/38/41,8/38/39,1/38/38,3/57/53,2/58,5/ $60,8 / 91,2 \mu$.

Indice antennaire : 1,00 (variant entre 0,94 et 1,1 selon les exemplaires).

Disposition des sensilles: 1 ou 2 sur le $8^{e}$ article, 2 sur le $9^{e}, 3$ sur le $10^{e}$, 1 sur le premier. Les autres en sont dépourvus.

Palpe : deux premiers articles cylindriques. Troisième article à peine renflé dans son tiers distal. Présence de plusieurs fossettes sensorielles.

Longueur des articles: 91,2/91,2/40/42 $\mu$ (deux premiers articles comptés ensemble).

Longueur de l'aile : 1,4 mm. ; largeur : 0,63 ; costa : 0,80 . 
Une spermathèque unique, tout à fait semblable à celle de $C$. stigma, de forme sphérique avec un appendice en forme de doigt de gant recourbé. Largeur : $49,4 \mu$.
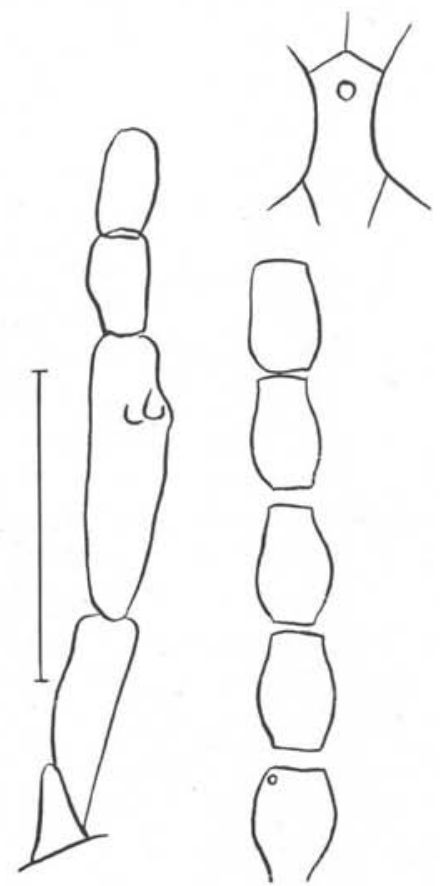

Fig. 13. - Culicoides stigmoides n. sp.; premiers articles antennaires et espace inter-oculaire de la femelle (même échelle que fig. 8).

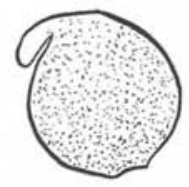

Fig. 14. - Culicoides stigmoides femelle. Spermathèque.

Epines du peigne tibial au nombre de 5 à 6 dont les longueurs sont : 64,6/ $45,6 / 45,6 / 41,8 / 34,2$ (pas de $6^{\circ}$ soie sur le type). 
Il est aisé de reconnaître les mâles de $C$. stigmoides à leur membrane spiculée. La diagnose entre les femelles de cette espèce et celles de $C$. stigma est actuéllement impossible.

Ardennes: La Ferée (source de la Serre, boues).

Ardennes belges: étang de Nimelette (berge).

Rappelons que nous avions signalé cette espèce sous le nom de $C$. stigma dans les terrains fangeux des Vosges:

Gresswiller et Hasslach dans le Bas-Rhin; Soultzeren dans le Haut-Rhin.

\section{Conclusion}

Nous décrivons dans cette étude quatre nouvelles espèces de Culicoides : C. clastrieri, trouvé en Seine-et-Marne et dans la plaine du Rhin, $C$. jurensis dans le Jura français, $C$. helveticus dans le Jura suisse et $C$. stigmoides dans les Ardennes et les Vosges. Les types sont déposés à l'Institut de Parasitologie de la Faculté de Médecine de Strasbourg. Nous avons placé ces espèces respectivement dans les groupes odibilis, cubitalis et stigma. Nous signalons pour la première fois en France $C$. lupicaris (dans les Ardennes).

A propos de l'étude des nouvelles espèces du groupe odibilis, nous réhabilitons $C$. pictimargo pour lequel nous avions proposé précédemment une mise en synonymie. Enfin, nous signalons des localisations nouvelles pour les espèces C. odibilis, C. musilator, C. cubitalis, C. pulicaris, C. punctatus, C. sphagnumensis var. vogesicus, $C$. pallidicornis, C. fascipennis, C. riethi (capturé à la lumière en Seine-et-Marne), $C$. obsoletus.

\section{BIBLIOGRAPHIE}

Arnaud (P.), 1956. - The Heleid genus Culicoides in Japan, Korea and Ryukyu Islands (Insecta : Diptera). Microentomol., 21, 84-207.

Austen (E. E.), 1921. - A contribution to knowledge of the blood-sucking Diptera of Palestine, other than Tabanidae. Bull. Ent. Res., 12, 107-

Austen (E. E.), 1912. - Notes on African Blood-sucking Midges (Familia Chironomidae, Subfamily Ceratopogoninae), with description of new species. Bull. Ent. Res. 3 : 99 (1).

Campbell (J. A.) et Pelham-Clinton (E. C.), 1960. - A taxonomic review of the British species of Culicoides Latreille (Diptera : Ceratopogonidae). Proc. R. Soc. Edinburgh (B), 67, 181-302.

(1) Que les lecteurs qui ont eu la courtoisie de ne pas nous faire rernarquer la grossière erreur que nous avions introduite dans le nom d'Austen (Krémer, Ann. Par. Hum. Comp., n. 1-2, 1961) veuillent bien trouver ici toutes nos excuses. Le Distinguished Service Order, dont fait partie le Major E. E. Austen, D.S.O., n'aurait en aucun cas dû être pris pour les initiales de ses prénoms. 
Callot (J.) et Kremer (M.), 1961. - Culicoides riouxi et Culicoides pseudohelio-philus, espèces nouvelles du groupe des Culicoides à ailes sans taches (Diptera, Ceratopogonidae). Ann. Par. Hum. Comp., 36, 677-688.

Carter (H. F.), Ingram (A.) et Macfie (J. W. S.), 1920. - Observations on the Ceratopogonine Midges of the Gold Coast with descriptions of new species. Part. II. Ann. Trop. Med. Par. 14, 211-274.

Clastrier (J.), 1957. - Notes sur les Cératopogonidés II. Quelques Culicoides d'Algérie à ailes tachetées. Arch. Inst. Past. Alg., 35, 404-444.

- 1961. - Notes sur les Cératopogonidés. XIV. Cératopogonidés de la Région éthiopienne (2). Arch. Inst. Past. Alg., 39, 302-337.

Downes (J. A.) et Ketrte (D. S.), 1952, - Description of three species of Culicoides Latreille (Diptera: Ceratopogonidae) new to Science, together with notes on, and a revised Key to the British Species of the Pulicaris and Obsoletus Groups. Proc. R. Ent. Soc. Lond. (B), 21, 61-78.

Edwands (F. W.), 1939. - Ceratopogonidae in \& Edwards, Oldroyd and Smart. British Blood-sucking Flies ». Brit. Mus. (Nat. Hist.), 25-50, et appendice, 129-148.

Kremer (M.) et Callot (J.), 1961. - Sur quelques Culicoides (Diptera Ceratopogonidae) du Haut-Valais. Acta Tropica (Bâle), 18, 354-356.

- - 1961. - Culicoides musilator n. sp. et espèces du groupe odibilis nouvelles pour la faune française (Diptera: Ceratopogonidae). Ann. Par. Hum. Comp., 36, 689-699.

- Contribution à l'étude des Cératopogonidés (Diptères : Nématocères) des terrains tourbeux des Hautes-Vosges. Monographie sur le Hohneck (sous presse).

Rioux (J.-A.), Descous (S.) et PeCH (J.), 1951. - Un nouveau Cératopogonide arboricole : Culicoides haranti n. sp. (Diptera Heleidae). Ann. Par. Hum. Comp., 34, 432-438.

Williams (R. W.), 1955. - Two new species of Culicoides from Cheboygau Country, Michigan (Diptera, Heleidae). Proc. Ent. Soc. Wash., 57, 269274 .

Faculté de Médecine de Strasbourg, Institut de Parasitologie (Directeur: Professeur J. Callot) 